\title{
The Application Research of Intelligent Identification Technology in Industrial Automation
}

\author{
Guigen Pan
}

Jiangxi Industry Vocational Technical College of Industry \& Trade, Nanchang, Jiangxi Province, China

Keywords: Smart technology, Identification technology, Industry, Automation

\begin{abstract}
With the support of science and technology, computer artificial intelligence recognition technology has made great progress, and it has also expanded the scope of applications in various development areas of society, promoting the development of human society. In the process of industrial automation production, the good use of intelligent identification technology can not only further improve the automation production efficiency and reduce production errors, but also can largely rely on the characteristics of intelligent manufacturing technology to improve production safety and make China's industrialization new. To this end, this paper explores the current application status of computer artificial intelligence recognition technology and considers the shortcomings in its application in order to provide a scientific direction for the development in industrial automation.
\end{abstract}

\section{Introduction}

Computer artificial intelligence technology mainly uses computers to simulate and learn human thinking, consciousness, and behavior, and then outputs instructions through computer programs. Among them, computer artificial intelligence recognition technology is an artificial intelligence technology that is widely used in human life. One, it has a great impact on our lives, so it has a broader development space than other artificial intelligence technologies, greatly improving the convenience of life and production. However, China's research on artificial intelligence recognition technology it started late, so there are still bottlenecks in many applications. Therefore, we need to carry out an in-depth analysis of the limitations and find the focus of future research in order to achieve a breakthrough in artificial intelligence recognition technology [1].

\section{Overview of Artificial Intelligence Recognition Technology}

The artificial intelligence recognition technology is mainly developed based on computer artificial intelligence technology. The research and development of this technology is to simulate and expand human intelligence. Through the analysis of the essence of intelligence, we have developed intelligent machines that are comparable to human intelligent reactions. In the field, the key technologies mainly include image recognition, speech recognition, robots, and natural language processing technologies, etc. [1]. In the process of artificial intelligence recognition technology, professional recognition devices are needed to intelligently identify the information of items to be identified. Get it and pass the obtained information to the computer processing system through the identification device. For example, during our shopping process, the barcode scanner used by the cashier is a type of intelligent identification device. The cashier uses the code scanning gun scan the product barcode to obtain the product name and price, and then automatically calculate the total price of the product by entering the quantity [2]. The entire process is the application process of artificial intelligence identification technology. It can be seen that the birth of artificial intelligence identification technology and application, greatly promote people's lives and production of automation and intelligence to improve the quality of life and productivity. 


\section{Classification of Artificial Intelligence Recognition Technology}

Computer artificial intelligence recognition technology is widely used in our lives and work. Through summary analysis, we can know that there are two major types in specific applications, mainly "inanimate" recognition and "alive" recognition.

\subsection{Smart Card Technology}

This technology mainly uses smart cards to realize automatic identification. In essence, smart cards are "integrated circuit cards" that can perform independent storage and calculation. There is a professional back-end computer system that also needs to complete information collection, transmission, management, and encryption [2]. This technology is widely used in daily life, such as vehicle high-speed ETC, item identification and so on.

\subsection{Barcode Recognition Technology}

This technology mainly identifies one-dimensional codes and two-dimensional codes. The two-dimensional code technology is based on the development of one-dimensional codes. It is more advanced than one-dimensional codes in terms of information capacity, information density, language character reality, and error correction capabilities [2]. From the current point of view, two-dimensional code identification has become the mainstream of life applications, and has gradually become an important information identifier for people, such as the Alipay two-dimensional code used for payment, WeChat two-dimensional code, movie ticket two-dimensional code, and so on.

\subsection{Radio Frequency Identification Technology}

This technology belongs to the non-contact automatic symbol recognition technology, which mainly reads and recognizes the target directly through wireless electromagnetic waves. In this process, the radio signal transmits the data information from the item label through the electromagnetic field, thereby achieving automatic identification and tracking effect [3]. At present, the main application of this technology and article identification, in the future development, may replace the currently widely used barcode technology, and become the best method for article identification management.

\subsection{Voice Recognition Technology}

This technology belongs to non-contact recognition technology. It mainly recognizes the user's voice and analyzes its audio, tone, and timbre data information. As the only feature of the user, sound can be directly used for technical operations without the use of hands and eyes other organs [4]. With the rapid development of modern technology, many voice recognition technologies and software have been developed and applied, and they can be seen in many fields.

\subsection{Face Recognition and Fingerprint Recognition Technology}

This technology is mainly an artificial intelligence recognition technology that analyzes facial features to achieve identity identification. During the work process, facial features can be tracked and stored as data, and the image and exposure intensity are automatically enlarged to achieve accurate identification [3]. The fingerprint recognition technology mainly uses the unique characteristics of human fingerprints to collect and store fingerprint texture information, and conducts comparison and analysis in the process of identifying users to determine the identity of people.

\section{Application Status of Computer Artificial Intelligence Recognition Technology}

Compared with foreign countries, computer artificial intelligence recognition technology started late in China. At present, the research on computer systems, programs, and software is not mature, but China's computer artificial intelligence recognition technology has developed rapidly and the 
research and development results have a high conversion rate. In the current application, it has gradually become an irreplaceable technological achievement in social development. Specifically, its application is mainly reflected in two aspects:

\subsection{Non-Living Technical Practice}

From the current stage of China's technical practice, it mainly includes three items: barcode identification technology, smart card technology, and radio frequency identification technology. Among them, the barcode identification technology is the most widely used. The implementation of QR codes and barcodes for apps such as WeChat are based on this technology the application of barcode recognition technology can centrally display the text information added to the information capacity, improve the information density, and have automatic error correction capabilities, which play an important role in improving the accuracy and efficiency of information identification and collection. The core of smart card technology is also intelligent automatic identification. It is an integrated circuit card with automatic, independent reserve and computing functions. When combined with a computer, it can realize its intelligent automatic information collection, processing, transmission, management and other functions. Technology has a wide range of applications in the physical field [5]. And the radio frequency identification technology, this technology through the non-contact automatic identification of symbols, can directly identify the information on the item by radio signals, can play an automatic identification and tracking function.

\subsection{Technological Practice with Life Characteristics}

Such as voice recognition technology, fingerprint recognition technology, face recognition technology and so on. Among them, the voice recognition technology also belongs to a non-contact recognition technology in the computer artificial intelligence recognition technology system. It takes sound as the main object and controls the operation by identifying the characteristics of the sound, such as the recognition of sound quality and tones. Currently, voice recognition technology is widely used in computer software and mobile terminal software programs, and has become a new type of control and recognition method. Fingerprint recognition technology uses fingerprint as a biological feature as a medium for identity recognition, which improves the security of identity recognition and is widely used in many precision verifications [4]. Face recognition technology is a recognition technology that uses facial visual characteristic information as a recognition medium. In the current stage of development, mobile phones and other identity verification links are widely used [5].

\section{Application of Intelligent Identification Technology in Industrial Automation}

\subsection{Human-Machine Operation}

With the help of intelligent identification technology, man-machine operations can be effectively completed. In the production process, manufacturing enterprises have higher requirements for the accuracy of products, and the use of intelligent technology can meet this requirement of manufacturing enterprises. For example, when manufacturing metal products, it is difficult to complete high-precision product production by relying only on manual labor. At this time, intelligent manufacturing methods are needed. By using numerical control equipment and computers, the accuracy of metal products can be improved [6]. In addition, the use of intelligent technology can quickly and accurately deal with problems that cannot be handled in the manufacturing process of machinery manufacturing. For example, when manufacturing high-precision measuring tools, intelligent technology robots can be used to perform comprehensive cutting and grinding of work pieces, thereby to improve the production efficiency and precision of high-precision measuring tools.

\subsection{Obtaining Information}

Intelligent identification can obtain information in industrial automation process control, and the process informationization in industrial production is relatively weak. With the development of industrial process automation, in order to achieve process informationization, intelligent 
identification is used to complete the information acquisition [5]. Intelligent identification solves the contradiction between industrial development and informatization. Intelligent identification can automatically obtain production information in industrial production, and store the production information in a database. In this way, information can be quickly collected, and the information collected at the same time invested in production and operation to meet the needs of industrial informatization. At the current stage of industrial production, the requirements for reducing the amount of labor are considered. Considering the needs of industrial production and construction, the application of intelligent identification should be implemented, the relevant content of information acquisition in intelligent identification should be tapped, and the committed information should be applied to industrial process control [6]. China's industrial automation process control has great development potential. After intelligent identification participation, industrial automation process control can not only improve production levels, but also cooperate with technical management to improve industrial automation production.

\subsection{System Modeling}

Intelligent identification in industrial automation process control is applied to system modeling. There are two specific applications for system modeling based on intelligent identification.

The first is data collection. Intelligent identification implements data collection in industrial process control. The counter records the number of pulses collected and stores them in the data register. The intelligent identification also uses A / D units in data collection, To achieve the conversion of analog to digital, the converted data will also be periodically stored in the register [7]. The intelligent identification is equipped with a printer device in the data collection, and regularly prints out various types of data for industrial process control. In the data acquisition, the PLC is connected to the communication module of the computer, and the stored data is read and processed by the computer. When the computer processes the running data, the PLC can be used as the computer data processing terminal. The second is production monitoring. Intelligent identification is applied to production monitoring in industrial automation process control. Production monitoring uses PLC to monitor the running status of various processes in the production process, at the same time to monitor the progress of industrial production, monitor abnormal information in industrial production, and record when abnormality or alarm information is detected, the intelligent recognition will automatically stop running the program when an abnormality is detected, or provide a solution for online adjustment. Intelligent identification in the production monitoring, the operation program is modified according to the actual situation of industrial automation process control, and the industrial operation counters and timers are mainly used to optimize industrial production [7].

\subsection{Dynamic Control}

Intelligent identification has a higher advantage in the dynamic control of industrial production. The dynamic control under the role of intelligent identification improves the management level of industrial production and helps improve the efficiency of industrial production. In recent years, dynamic control in industrial automation production has not only been applied to process processing, but also participates in management work to control the process of production more accurately [8]. Intelligent identification belongs to a class of emerging technologies. Intelligent identification provides conditions for dynamic control in industrial production, such as dynamic control in industrial production, and uses intelligent identification to connect industrial operating equipment in the industry and the PLC in the central control room to build stability between the two. The data communication path effectively connects the control system of industrial processing. Dynamic control under the function of intelligent identification, the central control room can directly control the process of industrial production, supervise the operation of industrial equipment, and send operable remote instructions to industrial production [8]. Common control instructions are: (1) UNI-TE network, PLC Build a communication mode with a touch-enabled operating station in the computer, and then use the UNI-TE network mode; (2) RS232 / RS485, this type of serial communication interface is used in the connection between PLC and smart instruments. 


\section{Conclusion}

The development of China's new era of industry is gradually developing towards the direction of complexity, large-scale, and integration. Industrial automation process control has become the main development content. The application of intelligent identification in industrial automation process control has changed the state of industrial operation and promoted the rational use of intelligent identification in industrial production lines. Intelligent identification simplifies the production process of industrial automation process control, and greatly improves the efficiency and effectiveness of industrial production. The application of intelligent identification is fully implemented in industrial automation process control, and the role of intelligent identification is exerted to optimize the operation process of industrial automation process control.

\section{References}

[1] B.B. Jiang, Application of Computer Artificial Intelligence Identification Technology, Technology and Innovation, vol.9, pp.153-154, 2018.

[2] X.Q. Xiong, Artificial Intelligence Identification Technology and Its Application, Information Communication, vol.1, pp.189-190, 2018.

[3] X.H. Wang, Application Bottleneck Analysis of Computer Artificial Intelligence Identification Technology, Science and Technology Innovation and Application, vol.8, pp.87-89, 2016.

[4] J.L. Wang, Exploration of the Application Bottleneck of Computer Artificial Intelligence Identification Technology, Science and Technology Outlook, vol.35, pp.93-94, 2016.

[5] L.J. Qin and X.J. Zhang, Research on Application of Artificial Intelligence Technology in Network Security Defense, Journal of Chifeng University (Natural Science Edition), vol.8, pp.55-56,2018.

[6] Q.F. Xu, Talking about the Modern Development of Machine Vision and Artificial Intelligence, Communication World, vol.9, pp.234-235, 2018.

[7] Ch.Y. Wang, Types and Applications of Computer Artificial Intelligence Identification Technology, Shandong Industrial Technology, vol.3, pp.149-151, 2019.

[8] J.X. Liu, Development and Application of Computer Artificial Intelligence Technology in Human Recognition, Global Market Information Review, vol.8, pp.242-244, 2018. 